\title{
MSc Med Bioethics and Health Law course for 2016
}

The Steve Biko Centre for Bioethics, at the University of the Witwatersrand in Johannesburg, will be offering a Masters course in bioethics and health law next year.The aim of the course is to train bioethics and medical law experts who will display skill and proficiency in the fields of bioethics and health law. The course aims to develop capacity and excellence in the ethical and legal analysis of issues arising in healthcare and research. Graduates will be able to apply their knowledge to the evaluation and management of bioethical and medico-legal problems and to propose solutions that are ethically acceptable and within the constraints of the law.

\section{Course structure}

The course is in two parts. Part 1, the coursework component, entails the successful completion of five taught units. Part 2 consists of a written research report of at least 15 000, but no longer than 20000 words in length. Part 1 constitutes $50 \%$ of the degree and Part 2 the remaining $50 \%$.

The units are taught in block release form. The block release for each is five days. After the teaching blocks have been completed, students are expected to produce written work in the form of short answer questions and essays. These must be submitted by the specified submission dates before the end of July. Successful completion of the units and the written research report will lead to the degree of Master of Science in Medicine in the field of Bioethics and Health Law. All the units are also offered as Certificate of Competency Courses.

\section{Brief description of the units (Part 1) Foundations of bioethics}

This unit is designed to enable you to analyse and evaluate issues in bioethics within the context of a solid ethical framework. Major theoretical, non-theoretical and other ways of viewing the world will be taught. The course intends to provide a foundational understanding of the relationship between concepts, logic and argumentation and fallacies of reasoning.

\section{Foundations of health law}

This unit will cover sources of South African law, the Constitution, statutory and common law in the context of health. Criminal, civil and family law and their interaction with health will be explored. The National Health Act will be discussed as well as the amendments to the Medicines Act. International law in relation to health is included in this unit.

\section{Advanced research ethics}

This unit introduces and explains a number of topical ethical issues in research ethics, including what constitutes unethical research, standards of care in a study, authorship guidelines and plagiarism. The role and modus operandi of Research Ethics Committees are outlined. Finally, clearly articulated standards of good clinical practice in research relevant to local realities and contexts are provided. These include obtaining valid informed consent, considering specific subgroups, standards of care for trial participants, access to study medications following completion of a clinical trial, issues pertaining to incentives affecting researchers and participants, releasing and publishing research results and the implementation of research findings.

\section{Advanced health ethics}

This unit addresses a number of important issues in bioethics and health law critically and in depth. Three to four important issues will be focused on each year relating to ethical and medico-legal issues in clinical contexts, reproductive health, policy and public health, resource allocation, genetics, health and human rights, environmental bioethics and others. Students will learn how to apply the ethical theories, ethical and legal principles, and their critical and analytical skills learnt in the foundations units to specific ethical and medico-legal questions.

\section{Research methods}

This course will provide students with rigorous training in independently designing, implementing and evaluating research, including research design, sampling procedures and data analysis. It will cover both research projects of an essentially normative and legal nature, as well as empirical projects (qualitative or quantitative) that include a normative and legal element. Students will be trained in every part of the research cycle (question formulation; literature review; use of secondary sources; primary and secondary argumentation; ethical and legal analysis; methodology to collect and analyse primary data), including the writing of reports. In terms of values and attitudes, the course aims to instil in students a commitment to ethical research. The course will prepare them to produce a research protocol/proposal suitable to their project, and ultimately a research report.

\section{Brief description of the research report (Part 2)}

Students apply what has been taught in the research methods course, as well as the other units in the programme, and carry out a supervised research study on an approved topic. This research project may be either essentially normative and legal in nature, or empirical with a normative and legal component.

\section{Course duration}

Fulltime students complete all five units and their research report in their first year of registration. Part-time students are advised to complete the four units 'Foundations of Bioethics', 'Foundations of Health Law', 'Advanced Research Ethics' and 'Advanced Health Ethics' in their first year. In their second year they should complete the 'Research Methods' Unit and their Research Report.

\section{Closing date for applications is 30 November 2015.}

For more information about the Centre and the MSc Med (Bioethics and Health Law) course, please visit the website: http://www.wits.ac.za/bioethics or contact Ms Jillian Gardner +27 11717 2719/2635. E-mail: Jillian.Gardner@wits.ac.za or Ms Tebogo Dithung +27 117172635 .

Email:Tebogo.Dithung@wits.ac.za 